Super I umi nal to subl umi nal transi ti on in the pul se propagati on i $n$ a resonant ly absorbi ng nedi um

\begin{tabular}{|l|l|}
\hline 著者 & $\begin{array}{l}\text { Tal ukder Ami nul, Anagi shi Yoshi mitsu, Tomi a } \\
\text { Nakot o }\end{array}$ \\
\hline $\begin{array}{l}\text { j our nal or } \\
\text { publ i cat i on ti t e }\end{array}$ & Physi cal Revi ew Let ter s \\
\hline vol une & 86 \\
\hline number & 16 \\
\hline page range & $3546-3549$ \\
\hline year & $2001-04$ \\
\hline 出版者 & Amer i can Physi cal Soci et y \\
\hline 権利 & (c) 2001 The Aner i can Physi cal Soci et y \\
\hline URL & ht t p: //hdl . handl e. net /10297/581 \\
\hline
\end{tabular}




\title{
Superluminal to Subluminal Transition in the Pulse Propagation in a Resonantly Absorbing Medium
}

\author{
Md. Aminul Islam Talukder, Yoshimitsu Amagishi, and Makoto Tomita \\ Department of Physics, Faculty of Science, Shizuoka University, 836 Ohya, Shizuoka 422-8529, Japan
}

(Received 20 October 2000)

\begin{abstract}
We have examined the propagation of femtosecond laser pulses in an absorbing dye solution through a short to a long range of propagation distance. The transmitted pulses show strong spectral shift and a superluminal to subluminal transition in the propagation velocity keeping its initial shape almost intact. It is verified that the peak velocity is well described by a modified group velocity $v_{S}$ defined within the framework of the saddle-point method as well as by a recent prediction of the net group delay of surviving frequency.
\end{abstract}

PACS numbers: $42.25 . \mathrm{Bs}$

For a century, the problems surrounding the pulse propagation through resonantly absorbing media have been leaving the physicists to puzzle over the meaning of group velocity. The study of light pulse propagation through dispersive media started with a series of papers by Sommerfeld and Brillouin [1,2]. They showed that the main signal propagates through the medium with a velocity always less than the light velocity $c$ in the vacuum, while the front edges of the precursors can travel at $c$. Garret and McCumber studied, both analytically and numerically, the propagation of a Gaussian shaped light pulse through a resonantly absorbing medium [3]. It was shown that, when the optical thickness is very small, the pulse propagation can be described by the conventional definition of group velocity. The remarkable consequence from their discussion is that, when the incident frequency lies in the anomalous dispersion region, the superluminal or even the negative pulse velocity can be possible. Although this conclusion seemed paradoxical at that time, it is asserted that the Poynting vector is always directed to the forward direction and none of the superluminal and negative velocities violate the causality $[3,4]$.

Since the concept of wave packets propagation is physically significant, the experimental verification of the meaning of propagation velocity is very important. Chu and Wong examined the pulse velocity in GaP:N with a laser tuned to the bound $A$-exciton line using a picosecond time-of-flight technique and observed that the pulse propagates through the sample with little distortion in the shape and with a velocity given by conventional definition of group velocity even when the velocity exceeds $c$ or becomes negative [5]. The propagation of optical pulses through one-dimensional photonic band gap materials $[6,7]$ and quantum controlled materials having a steep refractive index profile [8-11] has also attracted fundamental interest. It was found that the barrier traversal time of electromagnetic wave packets becomes independent of the barrier thickness [6]. The gain assisted superluminal pulse propagation through the lossless anomalous dispersion region $[12,13]$ and the ultraslow group velocity and enhanced nonlinear effects have been observed in coherently prepared absorptive and dispersive atomic gases [8-11].

It is true that the concept of the conventional group velocity $v_{g}$ is able to give a good picture of the pulse velocity in absorbing and gain media. However, it is limited to the thin sample only; i.e., it loses its meaning for a long propagation distance. In this Letter, we present our experimental investigation of the propagation of optical pulses through a resonantly absorbing dye solution from a short to a long distance. The meaning of the group velocity is discussed in terms of the propagation distance $z$ relevant to the absorption length $z_{\alpha}$, the spectral widths of the absorption line $\gamma$, and the incident laser pulses $\Delta \omega_{c}$. The experimental results are well described by a modified group velocity $v_{S}$, defined within the framework of the saddle-point method. It also verifies the Peatross et al. prediction that the concept of the conventional group velocity does not break down if applied to the surviving spectrum instead of the initial spectrum [14].

We used the optical pulses with a time duration $\Delta t_{p}=68 \mathrm{fs}$, spectral width $\Delta \omega_{c}=13.2 \mathrm{~nm}$, and with the $\Delta t_{p} \Delta \omega_{c}=2.6$ from a mode locked Ti:sapphire laser. The pulses were led into a Michelson interferometer to record the cross correlation signals between the incident and the transmitted pulses through the sample. A solution of IR125 in dimethyl sulfoxide (DMSO) in an optical cell of a thickness of $1 \mathrm{~mm}$ was inserted into one of the arms of the interferometer as the absorbing medium, while a reference cell containing the solvent only was inserted into another arm to compensate for the dispersion effects due to the glass cell and the solvent. The solid line in Fig. 1 shows the absorption spectrum of the sample dye measured with a scanning spectrophotometer, and the dotted line is the dispersion spectrum calculated using the Kramers-Kronig relation. In the pulse propagation through absorbing medium, the length scale relevant to the effective propagation distance is a product of the refractive index and propagation distance while the refractive index is linearly proportional to the dye concentration. To change 


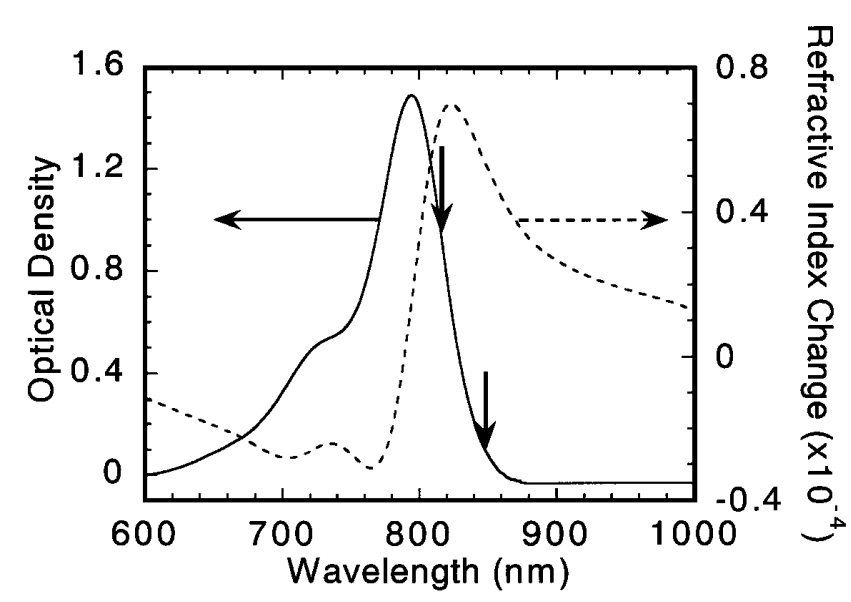

FIG. 1. Real and imaginary parts of the refractive index as a function of wavelength for the sample with a concentration of $4 \times 10^{-5} \mathrm{M}$. Vertical arrows indicate the wavelengths where the experiments are performed.

the effective propagation distance in our experiment, we controlled the dye concentration instead of the sample thickness. The path length in one of the arms of the interferometer was modulated with a piezoelectric transducer (PZT) at a frequency of $281 \mathrm{~Hz}$. The output interference signal was detected by a photomultiplier tube and fed into a lock-in amplifier with the reference signal from the PZT modulator. A reference autocorrelation trace was monitored simultaneously to find the exact position of the zero delay. The signal interference correlation trace was squared to average out the interference fringe patterns, and then the time delay at the pulse peak, $\tau_{\mathrm{ex}}$, was determined using a computational data processing routine. The spectrum of the transmitted pulse also was fed into a $25 \mathrm{~cm}$ spectrometer and recorded using a charge-coupled device camera.

Figure 1 shows the incident wavelengths of 816 and $849 \mathrm{~nm}$ which correspond to the anomalous dispersion region situated at the shoulder of the absorption line and the normal dispersion region situated at the tail of the absorption line, respectively. The observed time delays $\tau_{\mathrm{ex}}$ and the spectral peaks of the transmitted pulses are summarized in Fig. 2. In Fig. 2(a), with the incident pulses at $816 \mathrm{~nm}$, the peak position of the correlation trace moves toward the negative delay at the low concentration of dye indicating superluminal pulse propagation, and then turns back and moves toward the positive delay with increasing concentration of dye indicating subluminal pulse propagation. Figure 2(b) shows a monotonical increase in the positive delay in the normal dispersion region. It can be noted that the transmitted pulses show almost no changes to its initial shape. The dashed lines in Fig. 2 show the expected time delays if the pulse would have propagated with the conventional group velocity, $v_{g}$. We see that the conventional concept of the group velocity breaks down for thick absorbing medium. In all figures, the transmitted

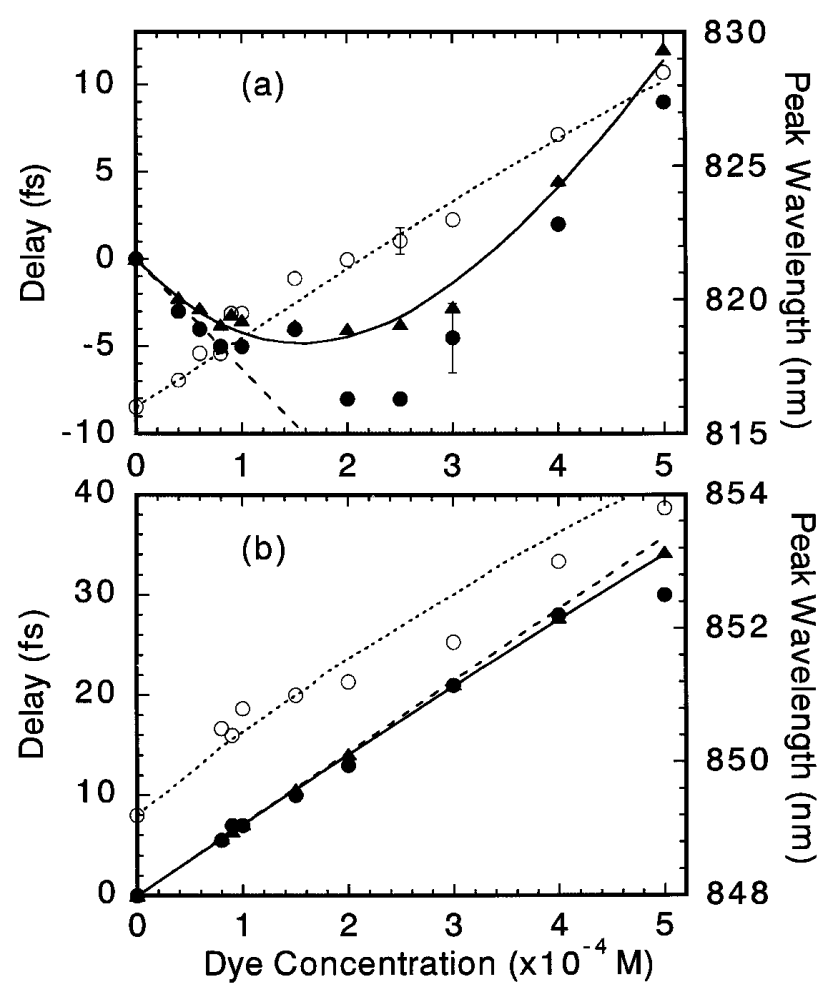

FIG. 2. Relative delays and peak wavelengths as a function of dye concentration for the incident pulses at (a) $816 \mathrm{~nm}$ and (b) $849 \mathrm{~nm}$. In all graphs, solid and open circles correspond to the experimental results for the delays $\tau_{\mathrm{ex}}$ and the peak wavelengths, respectively, while the dotted lines are calculated peak wavelengths. Dashed lines are the expected delays if the pulse would have propagated with $v_{g}$. Solid lines and solid triangles represent the expected delays $\tau_{S 1}$ and $\tau_{S 2}$, respectively. Vertical bars indicate the experimental errors.

pulses show the spectral redshift with increasing dye concentration. It would be worthwhile to note that the changes in the spectral widths of the transmitted pulses were very small. For the experiments with pulses of $816 \mathrm{~nm}$, the initial spectral width (FWHM) $13.2 \mathrm{~nm}$ becomes 13.3 and $14.2 \mathrm{~nm}$ through $8 \times 10^{-5}$ and $5 \times 10^{-4} \mathrm{M}$ of the sample belonging to superluminal and subluminal stage, respectively. Figure 3 shows the transmitted intensity as a function of propagation distance. It is seen that, in the normal dispersion region, the transmitted intensity shows an exponential damping. But, in the anomalous dispersion region, a nonexponential decay can be seen, especially for a short range of propagation distance in accordance with the spectral redshift, due to the strong absorption, as shown in Fig. 2.

The experimental results of the time delay as well as the transmitted pulse spectrum indicate that the conventional treatment of the group velocity fails rapidly with increasing propagation distance. Temporal profile of the pulses at a position $z, f(z, t)$, could be numerically calculated by the integration of the initial Fourier spectrum $A(\omega)$, with the frequency dependent phase delay associated with the propagation, 


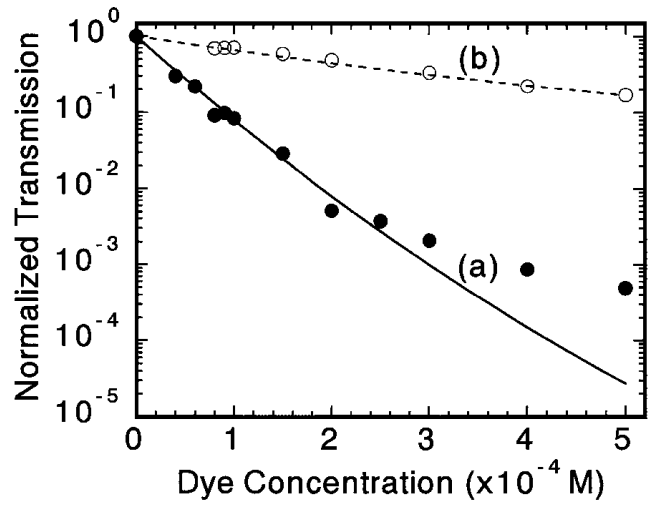

FIG. 3. Normalized transmission as a function of dye concentration for incident pulses at (a) $816 \mathrm{~nm}$ and (b) $849 \mathrm{~nm}$. The solid and open circles are the experimental results, while the solid and dashed lines are from the numerical calculation using Eq. (1).

$$
\begin{gathered}
f(z, t)=\int_{-\infty}^{\infty} d \omega \exp [P(\omega)], \\
P(\omega)=-i \omega\left(t-n(\omega) \frac{z}{c}\right)+\ln A(\omega),
\end{gathered}
$$

where $n(\omega)$ is the frequency dependent refractive index. In conventional treatment, the group velocity is introduced in the expansion of $n(\omega)$ as $\omega n(\omega)=\omega_{c} n\left(\omega_{c}\right)+$ $\left.\left(\omega-\omega_{c}\right)\{\partial \omega n(\omega) / \partial \omega\}\right|_{\omega_{c}}+\left(\frac{1}{2}\right)\left(\omega-\omega_{c}\right)^{2}\left\{\partial^{2} \omega n(\omega) /\right.$ $\left.\partial \omega^{2}\right\}\left.\right|_{\omega_{c}}+\ldots$, where $\omega_{c}$ is the central frequency of the incident pulse. When the spectral width of the incident pulse $\Delta \omega_{c}$ is narrow enough compared with that of the absorption line $\gamma$, as well as the propagation distance is short, the expansion converges very rapidly and can be terminated at the first order as $\left(\omega-\omega_{c}\right)^{2}\left\{\partial^{2} n(\omega) \omega / \partial \omega^{2}\right\}$ $(z / c) \ll 1$. The conventional definition of group velocity, $v_{g}=\left.\{\partial \omega / \partial k\}\right|_{\omega_{c}}$, then has a good meaning. If we employ the Lorentzian absorption line, $n(\omega)=$ $\left[1+\omega_{p}^{2} /\left(\omega_{0}^{2}-\omega^{2}-i \gamma \omega\right)\right]^{1 / 2}$, where $\omega_{0}$ is the resonant frequency and $\omega_{p}$ the plasma frequency, this criterion can be represented as $z \ll z_{2} \equiv z_{\alpha}\left(\gamma / \Delta \omega_{c}\right)^{2}$, which is not so restrictive as long as $\gamma / \Delta \omega_{c}>1$. In the experiments by Chu et al., $z /\left\{z_{\alpha}\left(\gamma / \Delta \omega_{c}\right)^{2}\right\} \sim 0.023$ [5] satisfies well the short propagation approximation.

In our case, using the femtosecond laser pulses with a broad spectrum, $z /\left\{z_{\alpha}\left(\gamma / \Delta \omega_{c}\right)^{2}\right\} \sim 0.438$, we observed the spectral shift in the transmitted pulse as shown in Fig. 2. The Fourier components in the shorter wavelength range experience stronger damping than those in the longer wavelength region and cause the spectral shift, $\delta \omega=\Delta \omega_{c}^{2} \operatorname{Im}[\partial n(\omega) \omega / \partial \omega](z / c)$. When $\delta \omega \sim \Delta \omega_{c}$, that is, $z>z_{1} \equiv z_{\alpha}\left(\gamma / \Delta \omega_{c}\right)$, the predominant frequency components contributing in the Fourier integration at $z$ are those which are drastically drifted during the pulse propagation. For $z_{1}<z<z_{2}$, it can occur that the conventional group velocity $v_{g}$ could properly describe the pulse velocity, even for the case of a considerable shift in the transmitted spectrum. In our study, the experiments done with the incident wavelength of $849 \mathrm{~nm}$ lie in this region for all examined dye concentration shown in Fig. 2, whereas the concentrations below $\sim 2 \times 10^{-5} \mathrm{M}$ are in this region for the incident pulses at $816 \mathrm{~nm}$. One may suggest the criterion at which the $v_{g}$ has good meaning as $\delta \tau \ll \tau$, where $\tau=z / v_{g}=(z / c)(\operatorname{Re}[\partial n(\omega) \omega / \partial \omega])$ and $\delta \tau=(\partial \tau / \partial \omega) \delta \omega$ is the change in the time delay due to the spectral dependent group velocity. This gives the propagation distance under which the $v_{g}$ is good for $z /\left\{z_{\alpha}\left(\gamma / \Delta \omega_{c}\right)^{2}\right\} \ll 1$, which essentially involves $z$ in terms of $z_{\alpha}, \gamma$, and $\Delta \omega_{c}$ is again the same condition as the second order can be ignored in Eq. (1).

As the velocity at which a pulse travels a distance $z$ is physically very important, there have been several proposals connected with the surviving spectrum to describe the pulse velocity beyond the short propagation and its narrow spectrum limits $[14,15]$. Tanaka et al. proposed a modified definition of the propagation velocity in which the path of integration in Eq. (1) is so chosen that it passes through the appropriate saddle points on the complex $\omega$ plane suppressing the oscillations of the integrand [15]. It has been pointed out that with an approximation, $\left|\partial^{2} P(\omega) / \partial \omega^{2}\right|_{\omega_{S}}=$ constant, the peak of the temporal profile of the pulse is determined by the minimum of $P\left(\omega_{S}\right), \partial P\left(\omega_{S}\right) / \partial t=0$. A significant result from this discussion is that, at $z$, the saddle point satisfies $\operatorname{Im}\left[\omega_{S}\right]=0$ at the time when the peak of the wave packet passes the point. With the definition of the saddle point,

$$
\begin{gathered}
\left.\operatorname{Re}\left[\frac{\partial \omega n(\omega)}{\partial \omega}\right]\right|_{\omega_{S}}-\frac{c t}{z}=0 \\
-\left.\operatorname{Im}\left[\frac{\partial \omega n(\omega)}{\partial \omega}\right]\right|_{\omega_{S}}+\left.\frac{c}{z} \frac{\partial \ln A(\omega)}{\partial \omega}\right|_{\omega_{S}}=0,
\end{gathered}
$$

one can obtain the velocity of the peak position as $v_{S}=$ $z / t=c /\left.\operatorname{Re}[\partial \omega n(\omega) / \partial \omega]\right|_{\omega_{S}}=\partial \omega /\left.\partial k\right|_{\omega_{S}}$ from Eq. (2), while the saddle points are obtained from Eq. (3). It is clear from Eq. (3) that, in the case with very weak absorption, $\omega_{S}$ equals the incident frequency $\omega_{c}$, and the pulse velocity is described by the conventional group velocity $v_{g}$. When absorption becomes strong, the saddle points move around on the complex $\omega$ plane following Eq. (3) with the complex $n(\omega)$ shown in Fig. 1. This corresponds to the observed spectral shift shown in Fig. 2. The solid lines in Fig. 2 show the time delays, $\tau_{S 1}$, expected from Eq. (2), where the saddle point frequencies $\omega_{S}$ are calculated using Eq. (3). Solid triangles in Fig. 2 show the time delays, $\tau_{S 2}$, expected from the relation of Eq. (2), where the saddle point frequencies $\omega_{S}$ are set to the peak positions of the observed transmitted spectra. The time delays $\tau_{S 1}$ and $\tau_{S 2}$, calculated on the basis of the saddle-point method, well describe the experimental results even where the $v_{g}$ fails completely, showing the superluminal to subluminal transition in the propagation velocity.

Recently, Peatross et al. have proposed an interesting definition of propagation velocity based on the time expectation integral over the Poynting vector as the arrival time of light pulse [14]. In their study, the time delay is 
separated into a sum of the net group delay and the reshaping delay due to absorption. This definition avoids the difficulty of approximations in the Fourier integration. As the reshaping delay before propagation for a well compressed pulse is quite small only the net group delay is relevant to describe the propagation velocity. The net group delay is simply the average of the group delay of each frequency component that survives the propagation through distance $z$,

$$
t=\frac{z}{c} \frac{\int d \omega|P(\omega)|^{2} \frac{\partial \operatorname{Re}[n(\omega) \omega]}{d \omega}}{\int d \omega|P(\omega)|^{2}} .
$$

Since $|P(\omega)|^{2}$ is dominated by $\omega_{S}$ in our case, this net group delay, Eq. (4), may be approximated as Eq. (2) in this paper as a special case.

At the high concentration region in Fig. 2(a), one may notice a very slight deviation between the delay, $\tau_{S 1}$, calculated from the saddle-point method and the experimentally observed delay $\tau_{\text {ex }}$. This discrepancy could be attributed to the approximation used in the present treatment of the saddle-point method which is based on the approximation in the expansion of $P(\omega)$ assuming $\left|\partial^{2} P(\omega) / \partial \omega^{2}\right|_{\omega_{s}}=$ constant. This approximation is valid when the spectral width of the incident pulse is narrow. We have demonstrated this point in Fig. 4 for a pulse propagating through a medium with a Lorentzian absorption line. The solid lines are time delays, $\tau_{N}$, calculated from the numerical integration of Eq. (1), while the dashed lines show $\tau_{S 1}$ calculated from the saddle-point method using Eqs. (2) and (3). When the spectral width is narrow, $\tau_{S 1}$ shows an excellent agreement with $\tau_{N}$ even for a very long propagation distance. In terms of the net group delay of Peatross et al. [14], this discrepancy could be explained as a slight failure of a single frequency $\omega_{S}$ to adequately represent the spectral average of the survived frequency components. The value of $\left|(z / 2 \pi c) \operatorname{Re}\left[\omega \partial^{2} n(\omega) / \partial \omega^{2}\right] \Delta \omega_{c}\right|_{\omega_{S}}$ corresponds to $\sim 2.5 \mathrm{fs}$ at a concentration of $2 \times 10^{-4} \mathrm{M}$ for the incident pulses at $816 \mathrm{~nm}$, provides a good estimation of the deviation between $\tau_{S 1}$ and $\tau_{N}$ in Fig. 4, and between $\tau_{S 1}$ and $\tau_{\text {ex }}$ in Fig. 2. In Fig. 4, we also see that the distance $z_{T}$, at which the propagation velocity changes form the superluminal to subluminal, can be estimated as $\left(\omega_{c}-\omega_{T}\right) / \delta \omega \sim 1, z_{T} \propto \Delta \omega_{c}^{-2}$, where $\omega_{T}$ is the frequency at which the refractive index curves shift from anomalous to normal dispersion region.

A similar effect in the propagation of Alfvén wave packets in a dissipative plasma with an anomalous dispersion relation has been reported [16]. The velocity of Alfvén wave is much slower than that of light, so the superluminal to subluminal transition is usually hard to be observed. The optical experiments presented here are then much more suitable for observing such a transition in an absorbing medium.

In conclusion, we have observed the superluminal to subluminal transition in the propagation velocity of op-

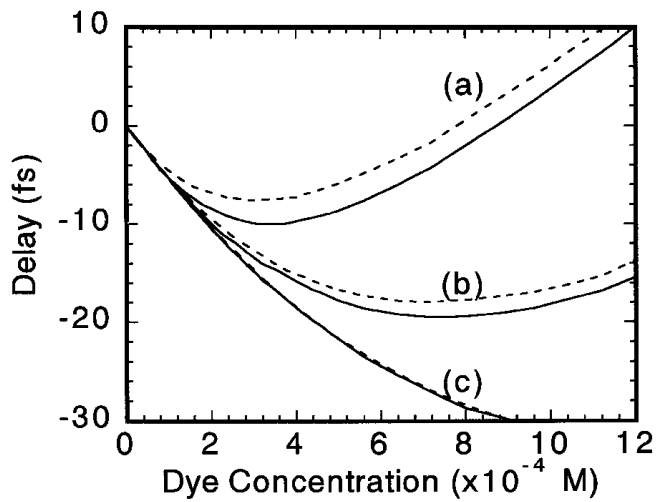

FIG. 4. Relative delay as a function of dye concentration for incident pulses at $816 \mathrm{~nm}$ with the spectral widths of $\gamma / \Delta \omega_{c}$ : (a) 4.8, (b) 7.3, and (c) 9.7. Solid lines are the calculated delays $\tau_{N}$, using Eq. (1), while the dashed lines express the delays $\tau_{S 1}$, expected from Eqs. (2) and (3).

tical pulses in an absorbing dye solution as a function of propagation distance. The observed peak velocity as well as the spectral shift in the transmitted pulses are well described by the modified group velocity $v_{S}$ defined within the framework of the saddle-point method. The results also verify the predictions of Ref. [14].

The authors are grateful to Professor M. Tanaka for fruitful discussions on the saddle-point method. This research was supported by Casio Science Foundation.

[1] A. Sommerfeld, Z. Phys. 8, 841 (1907).

[2] L. Brillouin, Wave Propagation and Group Velocity (Academic, New York, 1960).

[3] C. G. B. Garret and D.E. McCumber, Phys. Rev. A 1, 305-313 (1970).

[4] M. D. Crisp, Phys. Rev. A 4, 2104-2109 (1971).

[5] S. Chu and S. Wong, Phys. Rev. Lett. 48, 738-741 (1982).

[6] Ch. Spielmann, R. Szipöcs, A. Sting, and F. Krausz, Phys. Rev. Lett. 73, 2308-2311 (1994).

[7] A. M. Steinberg, P. G. Kwiat, and R. Y. Chiao, Phys. Rev. Lett. 71, 708-711 (1993).

[8] A. M. Akulshin, S. Barreiro, and A. Lezama, Phys. Rev. Lett. 83, 4277-4280 (1999).

[9] D. Budker, D.F. Kimball, S.M. Rochester, and V. V. Yashchuk, Phys. Rev. Lett. 83, 1767-1770 (1999).

[10] M. M. Kash, V. A. Sautenkov, A. S. Zibrov, L. Hollberg, G. R. Welch, M.D. Lukin, Y. Rostovtsev, E. S. Fry, and M. O. Scully, Phys. Rev. Lett. 82, 5229-5232 (1999).

[11] L. V. Hau, S.E. Harris, Z. Dutton, and C. H. Behroozi, Nature (London) 397, 594-598 (1999).

[12] L. J. Wang, A. Kuzmich, and A. Dogariu, Nature (London) 406, 277-279 (2000).

[13] R. Y. Chiao, Phys. Rev. A 48, R34-R37 (1993).

[14] J. Peatross, S. A. Glasgow, and M. Ware, Phys. Rev. Lett. 84, 2370-2373 (2000).

[15] M. Tanaka, M. Fujiwara, and Hideo Ikegami, Phys. Rev. A 34, 4851-4858 (1986).

[16] Y. Amagishi, H. Nakagawa, and M. Tanaka, Phys. Rev. E 50, 2217-2221 (1994). 